\title{
Overexpression of ETS-1 is associated with malignant biological features of prostate cancer
}

\author{
Bo Li $^{1,2}$, Yosuke Shimizu', Takashi Kobayashi ${ }^{1,3}$, Naoki Terada ${ }^{1}$, Koji Yoshimura ${ }^{1}$, Tomomi Kamba ${ }^{1}$, \\ Yoshiki Mikami ${ }^{4}$, Takahiro Inoue ${ }^{1}$, Hiroyuki Nishiyama ${ }^{5}$ and Osamu Ogawa ${ }^{1}$
}

E26 transformation-specific-1 (ETS-1), an ETS family transcription factor, has been reported to play an important role in a variety of physiological and pathological processes, but clinical implications of ETS-1 expression in prostate cancer (PCa), particularly high-risk cases, including response to androgen-deprivation therapy (ADT) have yet to be elucidated. We examined the expression of ETS-1 using immunohistochemical staining of paraffin-embedded prostate carcinoma tissue obtained by needle biopsy from 69 mostly advanced PCa patients. ETS-1 expression was compared with the clinicopathological characteristics of the 69 patients, including 25 who underwent ADT as a primary treatment. As a result, PCa patients with higher expression of ETS-1 were significantly more likely to be of high stage and high Gleason score $(P<0.05)$. There was no significant association between ETS-1 expression and the initial prostate-specific antigen (PSA) level. In the 25 patients treated by ADT, the staining score for ETS-1 was significantly associated with rapid development of castration-resistant disease within 24 months $(P<0.05)$, whereas the Gleason score and PSA level were not. In conclusion, increased ETS-1 expression was associated with a higher stage, higher Gleason score and shorter time to castration-resistant progression. These data suggest that immunostaining for ETS-1 could be a molecular marker for predicting a poor clinical outcome for PCa patients, particularly those with high-risk disease.

Asian Journal of Andrology (2012) 14, 860-863; doi:10.1038/aja.2012.107; published online 15 October 2012

Keywords: castration resistant; E26 transformation-specific-1 (ETS-1); immunohistochemistry; prostate cancer

\section{INTRODUCTION}

Prostate cancer (PCa) is the most commonly diagnosed malignancy among males in the developed countries and the second leading cause of cancer-related mortality. ${ }^{1}$ Patients with low-risk localized disease can be cured with surgery or radiation, whereas patients with high-risk or advanced disease have a poor prognosis. Although hormonal therapy in the form of medical or surgical castration can induce disease remissions even in these advanced cases, development of castrationresistant prostate cancer (CRPC) is eventually inevitable. In terms of treatment outcome, duration of the disease remission is critical for patient survival since therapeutic options for CRPC are limited and prognosis is poor, with a median survival of less than 2 years. ${ }^{2,3}$ However, there has been limited knowledge of pre-treatment prognostic factors for clinical outcome of high-risk or advanced PCa patients. ${ }^{4-6}$ Thus, there is an urgent need to establish new prognostic markers to aid in the prediction of unfavorable prognostic groups.

E26 transformation-specific-1 (ETS-1) was originally characterized as the v-ets retroviral gene, one of the two oncogenes ( $v$-myb and $v$-ets) in the avian leukemia retrovirus E26. ETS-1 has been reported to play an important role in a variety of physiological and pathological processes, including embryogenesis, wound healing and tumor progression, attributed to its ability to regulate the expression of proteins involved in angiogenesis, invasion and metastasis. ${ }^{7,8}$ It has been reported that ETS-1 is induced by some growth factor signals, including vascular endothelial growth factor and both acidic and basic fibroblast growth factors, ${ }^{9}$ and activates transcription of various metastasis-, angiogenesis- and invasion-associated genes, including genes encoding matrix metalloproteinases, urokinase-type plasminogen activator, and genes involved in energy metabolism. 7,10

In fact, previous studies showed close associations between ETS-1 expression in the primary tumor and local or metastatic progression of the tumor in human colorectal, ${ }^{11}$ breast, ${ }^{12,13}$ lung, ${ }^{14}$ gastric ${ }^{15,16}$ and ovarian cancers, ${ }^{17}$ indicating that ETS-1 expression is a useful marker for predicting metastasis and disease outcome in patients with malignancies. In terms of prostate tumor, a recent report showed that ETS-1 could be a tumor marker for the differentiation of latent prostatic carcinoma from clinically significant disease. ${ }^{18}$ The authors observed significant increases in ETS-1 expression along with progression through benign, latent and clinical PCa patients. They also reported that higher ETS-1 expression was associated with higher histological grade in both latent and clinical PCa tissues. A more recent report also showed a significant correlation between ETS-1 expression and histological grade of PCa. ${ }^{19}$ They also showed that ETS-1 activation can promote castration-resistant progression of PCa cells. However, the clinical significance of ETS-1 expression among relatively advanced PCa patients has yet to be elucidated. In the present study, we 
investigated the significance of ETS- 1 expression in the prediction of treatment outcome of advanced PCa patients.

\section{MATERIALS AND METHODS}

\section{Patients' characteristics}

Samples of prostatic needle biopsy were obtained from 69 Asian patients diagnosed with prostatic adenocarcinoma at Kyoto University Hospital, Kyoto, Japan. Clinical charts of the 69 patients were reviewed for their clinicopathological characteristics and most of the cases had a metastatic or high-risk disease as evident in Table 1. Mean ( \pm s.e.) age and prostatespecific antigen (PSA) were $72.4 \pm 1.0$ years and $428 \pm 197 \mathrm{ng} \mathrm{ml}^{-1}$, respectively. This study was approved by the Institutional Review Board of Kyoto University Graduate School of Medicine (G-52) and each patient provided a written informed consent for the use of their tissue samples.

There were 31 patients with metastatic disease, and 25 of them (the mean age at the time of biopsy was $74.6 \pm 7.7$ years) were available for follow-up data after androgen-deprivation therapy (ADT) based on goserelin acetate or leuprolide acetate. The 25 patients were additionally reviewed with regard to the time from the initiation of ADT to the development of CRPC, defined as three consecutive monthly increases in PSA against hormonal therapy including antiandrogens. The patients were followed until 31 May 2009, at the mean follow-up of 39.8 (s.e. 4.9) months. During this follow-up, 16 (64\%) patients developed CRPC within 24 months, while nine (36\%) were still responsive to $\mathrm{ADT}$ at the time of 24 months.

\section{Immunohistochemical examination}

Prostate biopsy specimens from the 69 patients were obtained before any kind of treatment. Paraffin-embedded sections were examined with immunohistochemical (IHC) staining. Material handling and immunostaining were performed according to methods described previously. ${ }^{18,20}$ Antigen retrieval was done with the microwave method. Briefly, citrate buffer ( $\mathrm{pH}$ 6.0) was boiled for $10 \mathrm{~min}$ in a pressure-proof rice cooker (Rakuchin Gozen; Daiya, Tokyo, Japan) by microwave. Glass slides were put into a metal rack and boiled for another $20 \mathrm{~min}$ in the rice cooker. Endogenous peroxidase activity was blocked by $0.3 \%$ $\mathrm{H}_{2} \mathrm{O}_{2}$ in methyl alcohol for $30 \mathrm{~min}$. ETS-1 (C-20) affinity-purified rabbit polyclonal antibody (Santa Cruz Biotechnology, Inc., Santa Cruz, CA, USA) was used at a dilution of $1: 500$. We confirmed, using western blotting, that the antibody recognizes ETS-1 but not ERG,

\section{Table 1 Clinicopathological characteristics of the 69 prostate cancer} patients

\begin{tabular}{ll}
\hline Variable & $\mathrm{n}(\%)$ \\
\hline Age (year) & \\
$\quad \leqslant 70$ & $27(39.1)$ \\
$\quad>70$ & $42(60.9)$ \\
Clinical stage & \\
$\quad$ Localized & $20(29.0)$ \\
Locally advanced & $18(26.1)$ \\
$\quad$ Metastatic & $31(44.9)$ \\
Gleason score & \\
$\quad 6-7$ & $40(58.0)$ \\
$8-10$ & $29(42.0)$ \\
Initial PSA (ng ml & \\
$\quad<10$ & \\
$10-20$ & $18(26.1)$ \\
$>20$ & $14(20.3)$ \\
\hline
\end{tabular}

Abbreviation: PSA, prostate-specific antigen.
ETV1 or ETV4 (data not shown). After overnight incubation with primary antibody at $4^{\circ} \mathrm{C}$, samples were then incubated with biotinylated antirabbit immunoglobulin G (Vector Laboratories, Burlingame, CA, USA) diluted to $1: 300$ for $40 \mathrm{~min}$. Avidin-biotin-peroxidase complex (ABC-Elite; Vector Laboratories, Burlingame, CA, USA) at a dilution of $1: 100$ was applied for $50 \mathrm{~min}$. The coloring reaction was carried out with $0.3 \mathrm{mg} \mathrm{ml}^{-1}$ of diaminobenzidine and $0.003 \% \mathrm{H}_{2} \mathrm{O}_{2}$ and nuclei were counterstained with hematoxylin. For negative control, the primary antibody was omitted.

The histopathological examination for ETS-1 expression and Gleason score was performed by two of the authors (Y Shimizu and Y Mikami), who were blinded to the clinicopathological data. ETS-1 expression levels were graded as 0,1 and 2 based on staining intensity to represent a range from no staining to strong staining.

\section{Statistical analyses}

Since immunohisochemical intensity was scored as categorical $(0,1$ or 2) and therefore those values did not follow the Gaussian distribution, the Pearson Chi-square test or Fisher's exact probability test was used to analyze the association between the expression of ETS-1 and Gleason score, tumor stage, patients' age and PSA level as appropriate. All statistical analyses were performed using SPSS 16.0 software (IBM, Armonk, NY, USA) and $P<0.05$ was considered statistically significant.

\section{RESULTS}

IHC for ETS-1 demonstrated signals both in the nucleus and cytoplasm of prostatic tumor cells but not in the adjacent benign tissues (Figure 1). There was a highly significant difference in the staining scores for ETS-1 among tumors at different clinical stage $(P=0.04$;

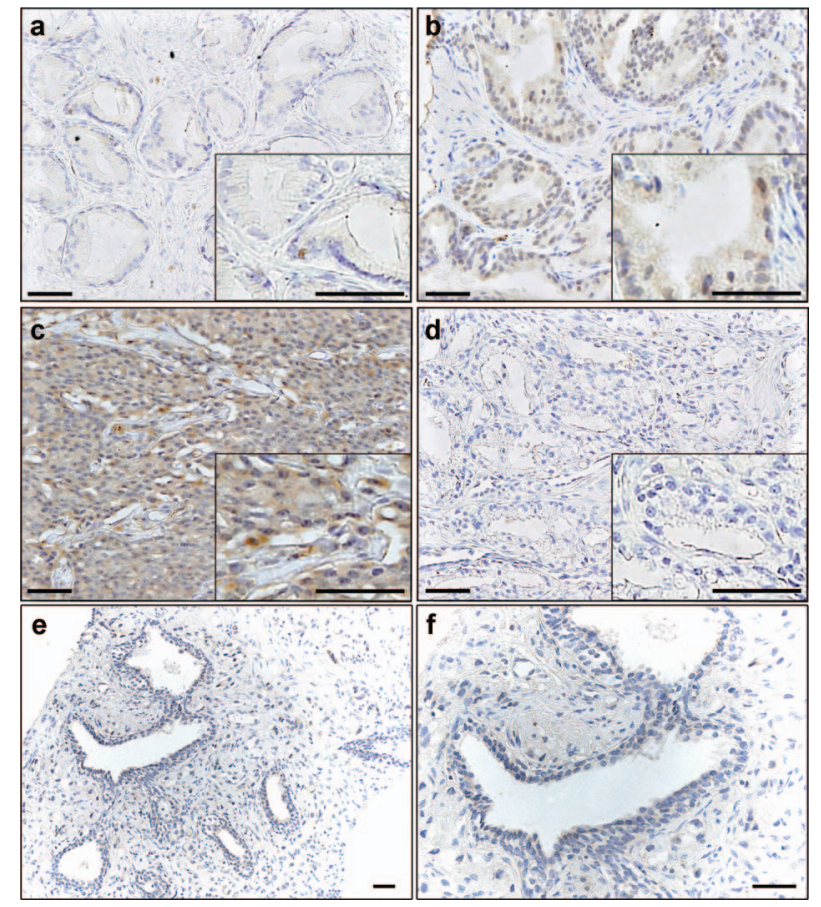

Figure 1 Immunohistochemistry of ETS-1 in prostatic carcinomas. It should be noted that heterogeneous expression of ETS-1 is observed both in the cytoplasm and the nucleus of the tumor cells. (a) No staining (staining score 0). (b) Weak staining (staining score 1). (c) Strong staining (staining score 2). (d) Negative control. Note that adjacent benign prostatic epithelia show negative stainings (e, f). Scale bars $=50 \mu \mathrm{m}$. EST-1, E26 transformation-specific- 1 . 
Table 2). Interestingly, eight (89\%) of the nine patients with a score of 2 had locally advanced or metastatic tumors. There was a statistically significant association among the staining scores for ETS- 1 and the tumor's Gleason score $(P=0.03)$. There was no significant association of the staining scores for ETS-1 with PSA levels $(P=0.11)$ and age $(P=0.91)$.

For the 25 patients with metastatic PCa treated by ADT, there was a significant difference in the staining scores for ETS-1 between patients with and without rapid progression to CRPC within 24 months $(P=0.02$; Table 3), but not in the Gleason score $(P=0.23)$ or initial PSA level $(P=0.36)$.

\section{DISCUSSION}

In the present study, IHC staining showed that ETS-1 expression was localized to both the nucleus and cytoplasm, which is consistent with previous reports. ${ }^{11-13}$ The reliability of immunohistochemical techniques for the purpose of examining ETS-1 expression was supported by the well-correlated results of in situ hybridization. ${ }^{11,12}$ These collectively indicate that the IHC technique used in the present study is a reliable and reproducible method for evaluating the expression of functional ETS-1 protein.

Using this technique, we have demonstrated that ETS- 1 expression is associated with clinical stage and the Gleason score of PCa. About $45 \%(13 / 29)$ of the PCa with no ETS-1 expression (staining score 0 ) were clinically localized tumors, whereas $83 \%$ (33/40) of those with higher expression (staining score 1-2) were advanced (Table 2). Additionally, all cases except one (97\%) of those with higher ETS-1 expression contained a high-grade cancer component (Gleason score 7 or more). Our findings strongly suggest that ETS- 1 expression is associated with the aggressive biological features of $\mathrm{PCa}$, and therefore, associated with the adverse clinicopathological characteristics of PCa.

Additionally, we have shown that ETS-1 expression is significantly associated with the rapid castration-resistant progression of $\mathrm{PCa}$ after $\mathrm{ADT}$. This is consistent with a previous report by Smith et al. ${ }^{19}$ showing that ETS-1 activation led PCa cells to a castration-resistant status. Our results suggest that ETS-1 expression can be a useful predictor for treatment outcome of patients with advanced PCa. A major clinical problem with hormonal therapy of advanced PCa is the pre-treatment prediction of time to castration-resistant progression.
Recently, Sim et al. ${ }^{21}$ reported that failure to attain a nadir PSA of $<1 \mathrm{ng} \mathrm{ml}^{-1}$ after treatment was the most important predictor of time to CRPC and a nadir PSA of $>2 \mathrm{ng} \mathrm{ml}^{-1}$ was a risk factor for poor overall survival. However, those findings will be obtained only after treatment has been initiated, and as the authors reported, prediction of treatment outcome at pre-treatment settings was difficult since the biopsy Gleason score or initial PSA level was not a predictor of rapid progression to CRPC. Indeed, the biopsy Gleason score and initial PSA level also failed to predict time to progression to CRPC in the present study. In this regard, a significant association between increased ETS-1 expression and a shorter time to CRPC observed in this study would enable us to separate those patients who are likely to fail on hormone therapy from those who are more likely to achieve long-term stable disease at pre-treatment settings. This is an important prospect, which should be validated with a larger study in the future. On the other hand, we did not observe a significant association between expression of ETS- 1 and survival of patients with metastatic disease. Our interpretation is that although time to progress to CRPC is one of the determining factors of patient survival, there are several other factors that affect patient survival. In particular, recently, options for the treatment of CRPC patients have become available: for example, docetaxel. ETS-1 expression may be unrelated to response to docetaxel treatment. However, it is still important to predict time to progression to CRPC.

Aberrant activation of ETS family of transcription factors by gene fusion with certain androgen-dependent genes is a frequently observed alteration inducing invasion of PCa cells, ${ }^{22-24}$ which is now considered as an initiation ${ }^{25}$ and progression ${ }^{26}$ event in the natural history of PCa. Considering that members of the ETS family transcription factors share a number of target genes, ETS-1 is likely to play an important role in PCa. Indeed, a number of genes that can facilitate cancer progression are known as transcriptional target of ETS-1. ${ }^{7,10}$ Additionally, ETS-1 has been reported to activate transcription of Sp1 resulting in an increase in lipogenesis and cell proliferation through induction of fatty acid synthase, ${ }^{27}$ which is thought to be associated with castration-resistant progression of $\mathrm{PCa} .{ }^{28}$ Moreover, considering less frequent ERG gene fusion in PCa patients in Asian population, ${ }^{24}$ impact of overexpression ETS-1 could be more significant in this ethnic group.

We recognize that this study has a limitation in that it followed relatively few patients, although its strengths included utilization of

Table 2 Expression of ETS-1 in relation to clinicopathological characteristics

\begin{tabular}{|c|c|c|c|c|c|}
\hline Variable & $N$ & \multicolumn{3}{|c|}{ Case No. in different group of ETS-1 expression score } & $P$ \\
\hline \multicolumn{6}{|l|}{ Clinical stage } \\
\hline Locally advanced & 18 & $8(44)$ & $6(33)$ & $4(22)$ & \\
\hline Metastatic & 31 & $8(26)$ & $19(61)$ & $4(13)$ & \\
\hline \multicolumn{6}{|l|}{ Gleason score } \\
\hline $8-10$ & 29 & $5(17)$ & $19(66)$ & $5(17)$ & \\
\hline \multicolumn{6}{|l|}{ Age (years) } \\
\hline$\leqslant 70$ & 27 & $12(44)$ & $12(44)$ & $3(11)$ & 0.91 \\
\hline$>70$ & 42 & $17(40)$ & $19(45)$ & $6(14)$ & \\
\hline \multicolumn{6}{|l|}{ Initial PSA (ng ml ${ }^{-1}$ ) } \\
\hline$<10$ & 18 & $8(44)$ & $8(44)$ & $2(11)$ & 0.11 \\
\hline
\end{tabular}

Abbreviations: ETS-1, E26 transformation-specific-1; PSA, prostate-specific antigen. 
Table 3 Association between ETS-1 expression and the time to CRPC in 25 metastatic prostate cancer patients treated by ADT

\begin{tabular}{|c|c|c|c|c|}
\hline \multirow[t]{2}{*}{ Variable } & \multirow[t]{2}{*}{$N$} & \multicolumn{2}{|c|}{$\begin{array}{c}\text { Case No. in different group of } \\
\text { time to CRPC }\end{array}$} & \multirow[t]{2}{*}{$\mathrm{P}$ value } \\
\hline & & $\begin{array}{l}>24 \\
\text { months }\end{array}$ & $\begin{array}{l}<24 \\
\text { months }\end{array}$ & \\
\hline
\end{tabular}

\begin{tabular}{lrrrr}
\hline $\begin{array}{l}\text { ETS-1 expression } \\
\text { (score) }\end{array}$ & & & & \\
0 & 10 & 7 & 3 & 0.02 \\
1 & 11 & 2 & 9 & \\
2 & 4 & 0 & 4 & \\
Gleason score & & & & \\
6 or less & 2 & 2 & 0 & 0.23 \\
7 & 8 & 2 & 6 & \\
$8-10$ & 15 & 5 & 10 & \\
Initial PSA $\left(\mathrm{ng} \mathrm{ml}^{-1}\right)$ & & & & \\
$\quad<500$ & 19 & 8 & 11 & 0.36 \\
$>500$ & 6 & 1 & 5 & \\
\hline
\end{tabular}

Abbreviations: ADT, androgen-deprivation therapy; CRPC, castration-resistant prostate cancer; ETS-1, E26 transformation-specific-1; PSA, prostate-specific antigen.

pre-treatment biopsy specimens of advanced PCa patients, comprehensive data collection, analysis by a central pathologist and a reasonable duration of follow-up, which helped to compensate for the small number of samples. Prospective validation studies on ETS-1 expression together with other molecular markers are warranted in the future. It would also be interesting to elucidate other biomarkers that are potentially related to ETS-1 expression or which strengthen the predictive accuracy for treatment responses of advanced PCa patients in future studies. In this regard, it would be interesting to know the correlation of PTEN status with ETS-1 expression, given the recent findings about the causal relationship of increased AKT activity and elevated ETS-1 expression in PCa cell lines. ${ }^{19}$ Using biopsy tissues can be problematic as tumors often exhibit heterogeneity; however, clinical decision-making is often based on biopsy specimens. Since our patients were relatively advanced, the value of ETS-1 expression was unclear in relatively early cases such as those with PSA levels $<4.0 \mathrm{ng} \mathrm{ml}^{-1}$. It has been reported previously that the antibody used in this study can cross-react with Annexin V, an unrelated protein. ${ }^{29}$ Although we cannot strictly exclude the possibility that the antibody recognizes an unrelated protein, ETS-1 expression evaluated with the antibody still has diagnostic significance in predicting time to castration-resistant progression.

In conclusion, immunohistochemical analysis of PCa specimens clearly demonstrated that the expression of ETS-1 was associated with the pathological grade and clinical stage of the disease. Importantly, we found that ETS-1 expression was significantly associated with time to CRPC in patients with advanced PCa. Although further systematic studies are necessary, these findings support the possibility that a higher expression of ETS-1 can play an important role in the poor differentiation of PCa cells. Thus, ETS-1 may be a useful marker for the risk of PCa progression.

\section{AUTHOR CONTRIBUTIONS}

$\mathrm{BL}, \mathrm{YS}$ and TK designed the experiments and $\mathrm{BL}$ and YS carried out the experiments. YS and YM evaluated the IHC. BL, TK, NT, KY, TK, TI and $\mathrm{HN}$ analyzed and discussed the data. $\mathrm{HN}$ and $\mathrm{OO}$ supervised the project. BL, YS and TK wrote the manuscript.

\section{COMPETING FINACIAL INTERESTS}

All authors declare that there are no competing financial interests.

\section{ACKNOWLEDGMENTS}

This study was partly supported by a grant-in-aid from the Ministry of Education, Culture, Sports, Science, and Technology of Japan, Takeda Science Foundation (to B Li), and the Uehara Memorial Foundation (to T Kobayashi and O Ogawa).

1 Siegel R, Ward E, Brawley O, Jemal A. Cancer statistics, 2011: the impact of eliminating socioeconomic and racial disparities on premature cancer deaths. $C A$ Cancer J Clin 2011; 61: 212-36.

2 Tannock IF, de Wit R, Berry WR, Horti J, Pluzanska A et al. Docetaxel plus prednisone or mitoxantrone plus prednisone for advanced prostate cancer. N Engl J Med 2004; 351: 1502-12.

3 Petrylak DP, Tangen CM, Hussain MH, Lara PN Jr, Jones JA et al. Docetaxel and estramustine compared with mitoxantrone and prednisone for advanced refractory prostate cancer. N Engl J Med 2004; 351: 1513-20.

4 Lu-Yao GL, Albertsen PC, Moore DF, Shih W, Lin Y et al. Survival following primary androgen deprivation therapy among men with localized prostate cancer. JAMA2008; 300: 173-81.

5 DiBlasio CJ, Malcolm JB, Hammett J, Wan JY, Aleman MA et al. Survival outcomes in men receiving androgen-deprivation therapy as primary or salvage treatment for localized or advanced prostate cancer: 20-year single-centre experience. BJU Int 2009; 104: 1208-14.

6 He J, Zeng ZC, Yang P, Chen B, Jiang W et al. Clinical features and prognostic factors for patients with bone metastases from prostate cancer. Asian J Androl 2012; 14: 505-8.

7 Dittmer J. The biology of the Ets 1 proto-oncogene. Mol Cancer 2003; 2: 29.

8 Oikawa T. ETS transcription factors: possible targets for cancer therapy. Cancer Sci 2004; 95: 626-33.

9 Iwasaka $\mathrm{C}$, Tanaka K, Abe M, Sato Y. Ets-1 regulates angiogenesis by inducing the expression of urokinase-type plasminogen activator and matrix metalloproteinase-1 and the migration of vascular endothelial cells. J Cell Physiol 1996; 169: 522-31.

10 Verschoor ML, Wilson LA, Verschoor CP, Singh G. Ets-1 regulates energy metabolism in cancer cells. PLoS One 2010; 5: e13565.

11 Nakayama T, Ito M, Ohtsuru A, Naito S, Sekine I. Expression of the ets-1 protooncogene in human colorectal carcinoma. Mod Pathol 2001; 14: 415-22.

12 Behrens $\mathrm{P}$, Rothe M, Wellmann A, Krischler J, Wernert N. The Ets-1 transcription factor is up-regulated together with MMP 1 and MMP 9 in the stroma of pre-invasive breast cancer. J Pathol 2001; 194: 43-50.

13 Buggy Y, Maguire TM, McGreal G, McDermott E, Hill AD et al. Overexpression of the Ets-1 transcription factor in human breast cancer. Br J Cancer 2004; 91: 1308-15.

14 Sasaki H, Yukiue H, Moiriyama S, Kobayashi Y, Nakashima Y et al. Clinical significance of matrix metalloproteinase-7 and Ets-1 gene expression in patients with lung cancer. J Surg Res 2001; 101: 242-7.

15 Tsutsumi S, Kuwano H, Nagashima N, Shimura T, Mochiki E et al. Ets-1 expression in gastric cancer. Hepatogastroenterology 2005; 52: 654-6.

16 Yu Y, Zhang YC, Zhang WZ, Shen LS, Hertzog P et al. Ets1 as a marker of malignant potential in gastric carcinoma. World J Gastroenterol 2003; 9: 2154-9.

17 Takai N, Miyazaki T, Nishida M, Nasu K, Miyakawa I. c-Ets1 is a promising marker in epithelial ovarian cancer. Int J Mol Med 2002; 9: 287-92.

18 Alipov G, Nakayama T, Ito M, Kawai K, Naito S et al. Overexpression of Ets-1 protooncogene in latent and clinical prostatic carcinomas. Histopathology 2005; 46: 202-8.

19 Smith AM, Findlay VJ, Bandurraga SG, Kistner-Griffin E, Spruill LS et al. ETS1 transcriptional activity is increased in advanced prostate cancer and promotes the castrate-resistant phenotype. Carcinogenesis 2012; 33: 572-80.

20 Inoue T, Segawa T, Shiraishi T, Yoshida T, Toda Y et al. Androgen receptor, Ki67, and p53 expression in radical prostatectomy specimens predict treatment failure in Japanese population. Urology 2005; 66: 332-7.

$21 \mathrm{Sim}$ HG, Lau WK, Cheng CW. Predictors of androgen independence in metastatic prostate cancer. BJU Int 2004; 93: 1221-4.

22 Tomlins SA, Rhodes DR, Perner S, Dhanasekaran SM, Mehra R et al. Recurrent fusion of TMPRSS2 and ETS transcription factor genes in prostate cancer. Science 2005; 310: $644-8$

23 Sreekumar A, Poisson LM, Rajendiran TM, Khan AP, Cao Q et al. Metabolomic profiles delineate potential role for sarcosine in prostate cancer progression. Nature 2009, 457: 910-4.

24 Magi-Galluzzi C, Tsusuki T, Elson P, Simmerman K, LaFargue C et al. TMPRSS2ERG gene fusion prevalence and class are significantly different in prostate cancer of Caucasian, African-American and Japanese patients. Prostate 2011; 71: 489-97.

25 Tomlins SA, Laxman B, Dhanasekaran SM, Helgeson BE, Cao X et al. Distinct classes of chromosomal rearrangements create oncogenic ETS gene fusions in prostate cancer. Nature 2007; 448: 595-9.

26 Carver BS, Tran J, Chen Z, Carracedo-Perez A, Alimonti A et al. ETS rearrangements and prostate cancer initiation. Nature 2009; 457: E1; discussion E2-3.

27 Lu S, Archer MC. Sp1 coordinately regulates de novo lipogenesis and proliferation in cancer cells. Int J Cancer 2010; 126: 416-25.

28 Segawa T, Nau ME, Xu LL, Chilukuri RN, Makarem M et al. Androgen-induced expression of endoplasmic reticulum (ER) stress response genes in prostate cancer cells. Oncogene 2002; 21 : 8749-58.

29 Dittmer J. Does a truncated form of the transcription factor Ets 1 exist in breast cancer cells? Br J Cancer 2006; 94: 176-7. 